\title{
SUPPLEMENTARY MATERIAL: Ensemble MCMC: Accelerating Pseudo-Marginal MCMC for State Space Models using the Ensemble Kalman Filter
}

Christopher Drovandi ${ }^{*},+,, \|$, Richard G Everitt ${ }^{\S}$ Andrew Golightly ${ }^{\llbracket}$ and Dennis Prangle

\section{Appendix A: Correlated and unbiased eMCMC results for the population ecology example}
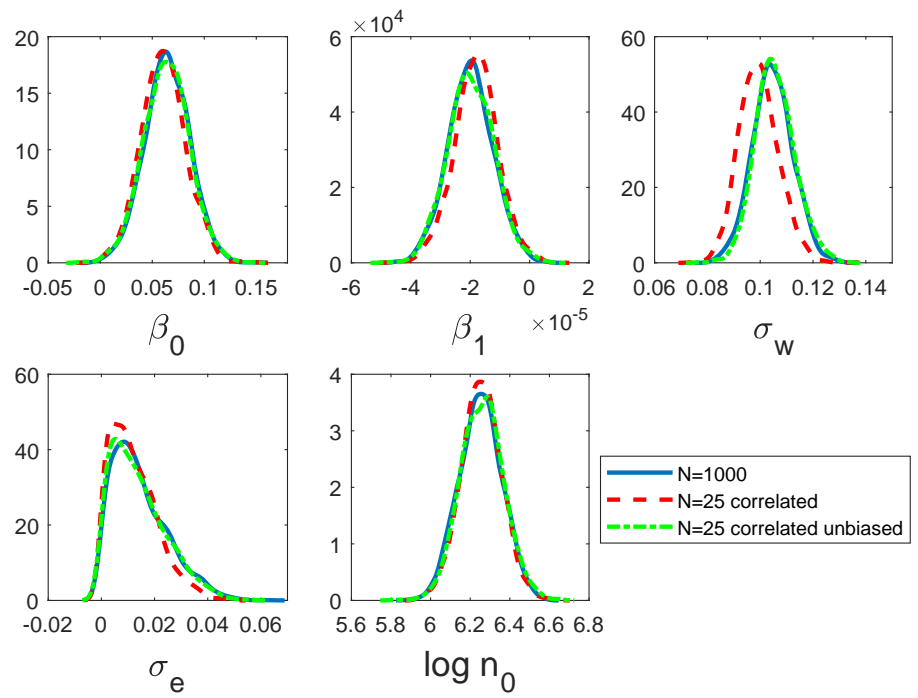

Figure 1: Approximate univariate posteriors for the Ricker model using eMCMC with $N=1000$ (blue solid), correlated eMCMC with $N=25$ (red dash) and correlated ueMCMC with $N=25$ (green dash-dot).

\footnotetext{
*School of Mathematical Sciences, Queensland University of Technology, Australia c.drovandi@qut.edu.au

${ }^{\dagger}$ ARC Centre of Excellence for Mathematical \& Statistical Frontiers (ACEMS)

$\ddagger_{\mathrm{QUT}}$ Centre for Data Science

$\S$ Department of Statistics, University of Warwick, UK richard.everitt@warwick.ac.uk

ISchool of Mathematics, Statistics and Physics, Newcastle University, UK Andrew.Golightly@newcastle.ac.uk Dennis.Prangle@newcastle.ac.uk

" Ordering of authors is alphabetical
} 

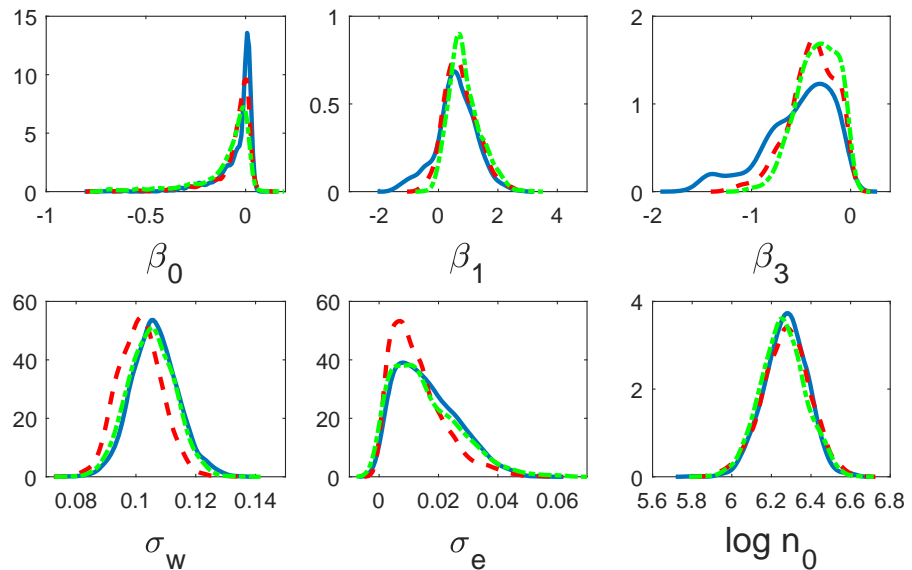

\section{$\mathrm{N}=1000$
$--\mathrm{N}=25$ correlated}

$---=\mathrm{N}=25$ correlated unbiased

Figure 2: Approximate univariate posteriors for the Ricker model using eMCMC with $N=1000$ (blue solid), correlated eMCMC with $N=25$ (red dash) and correlated ueMCMC with $N=25$ (green dash-dot).
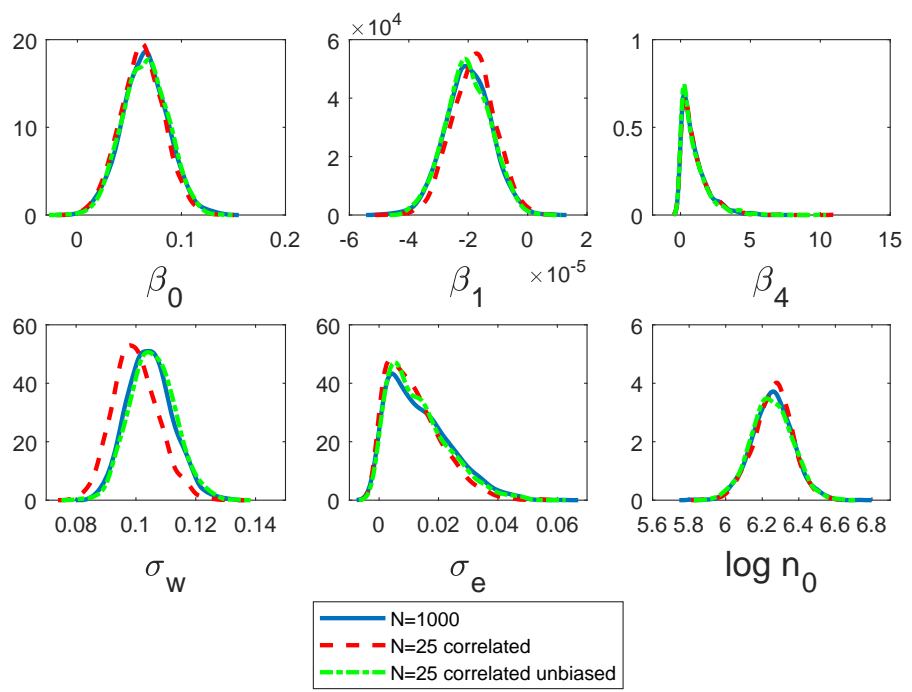

$\log n_{0}$

Figure 3: Approximate univariate posteriors for the Ricker model using eMCMC with $N=1000$ (blue solid), correlated eMCMC with $N=25$ (red dash) and correlated ueMCMC with $N=25$ (green dash-dot). 

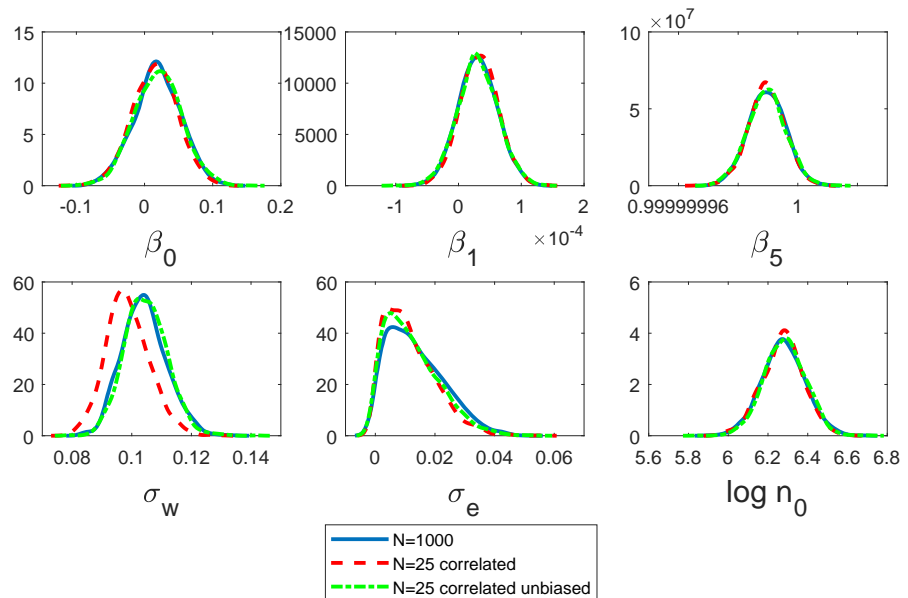

Figure 4: Approximate univariate posteriors for the Ricker model using eMCMC with $N=1000$ (blue solid), correlated eMCMC with $N=25$ (red dash) and correlated ueMCMC with $N=25$ (green dash-dot).

\section{Appendix B: RQMC eMCMC results for the population ecology example}
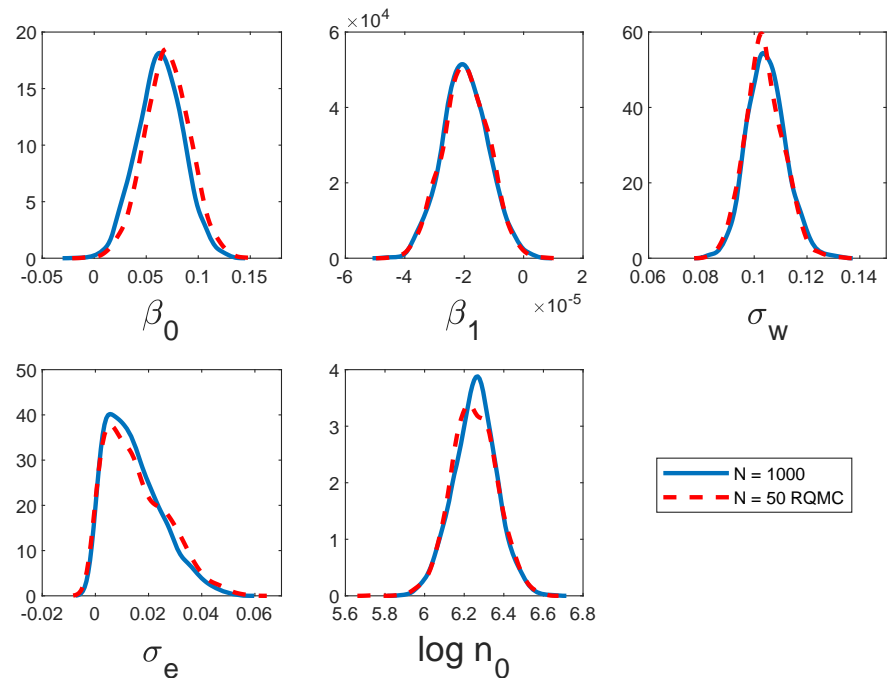

Figure 5: Approximate univariate posteriors for the Ricker model using eMCMC with $N=1000$ (blue solid) and rqme eMCMC with $N=50$ (red dash). 

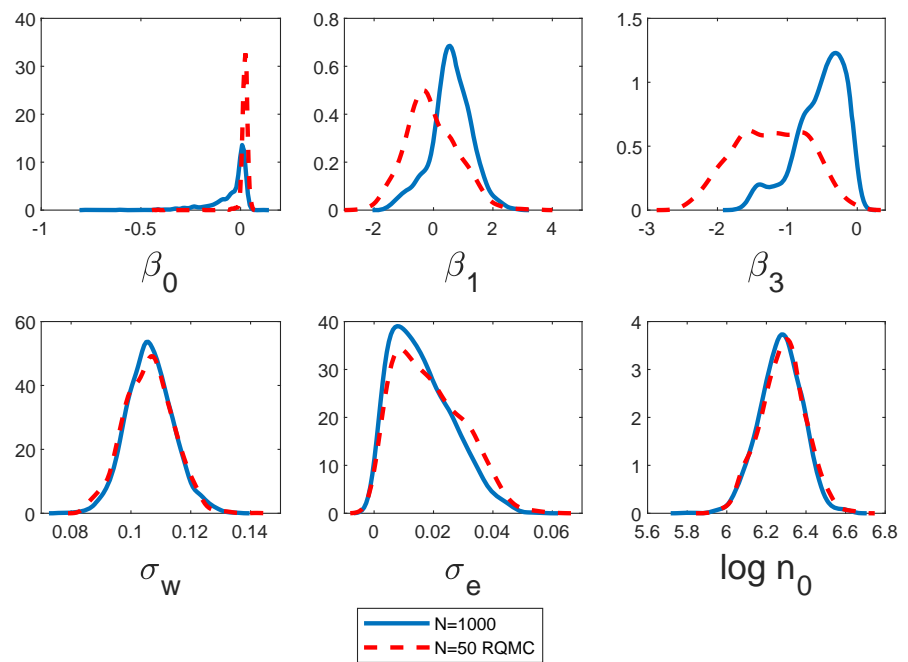

$\log n_{0}$

Figure 6: Approximate univariate posteriors for the theta-logistic model using eMCMC with $N=1000$ (blue solid) and rqme eMCMC with $N=50$ (red dash).
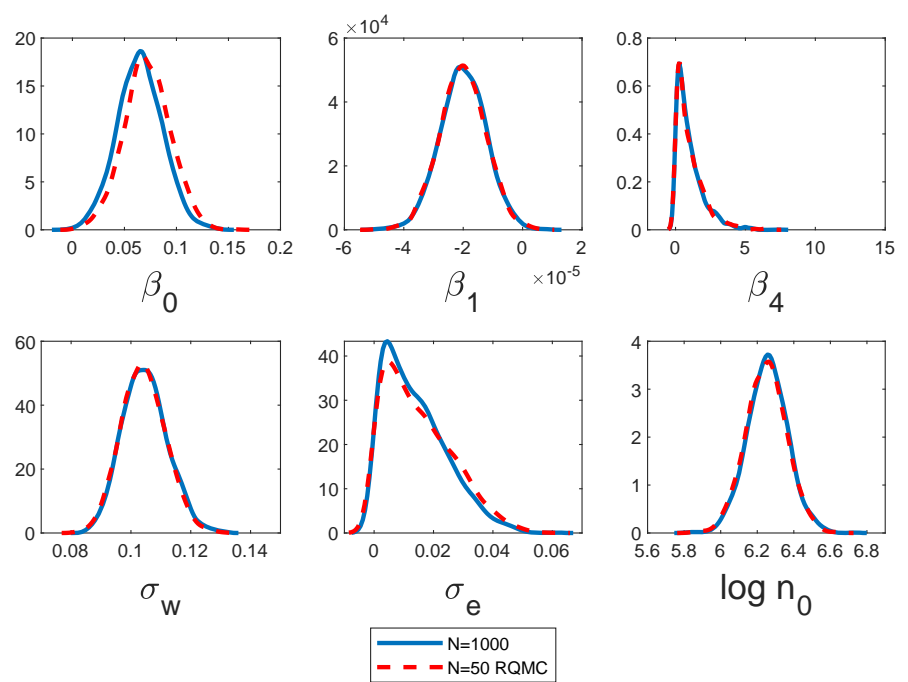

Figure 7: Approximate univariate posteriors for the mate-limited model using eMCMC with $N=1000$ (blue solid) and rqme eMCMC with $N=50$ (red dash). 

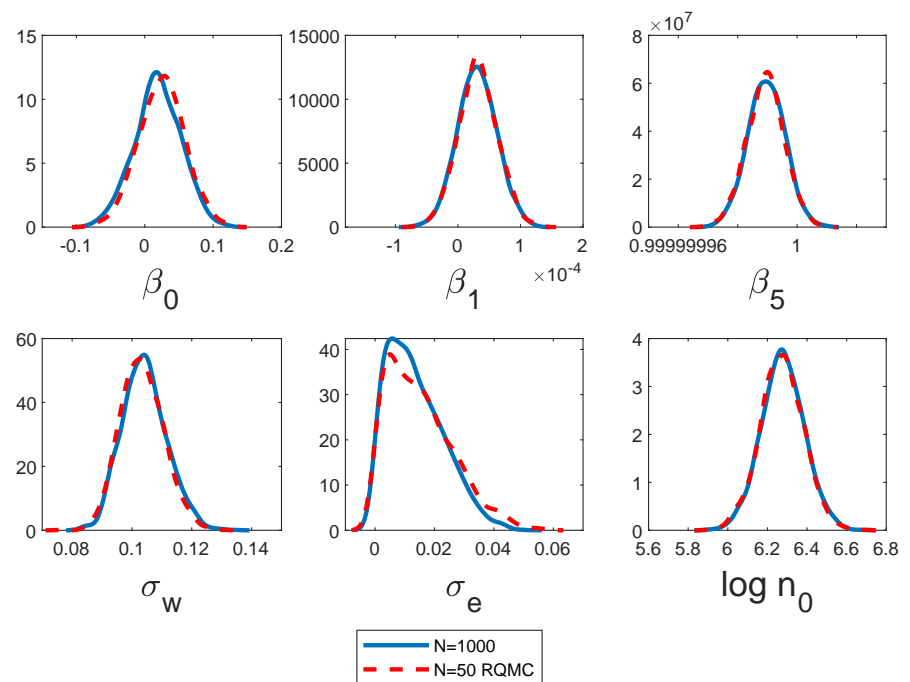

Figure 8: Approximate univariate posteriors for the theta-logistic model using eMCMC with $N=1000$ (blue solid) and rqme eMCMC with $N=50$ (red dash).

\section{Appendix C: Log likelihood estimate plots for Lorenz 63 example}
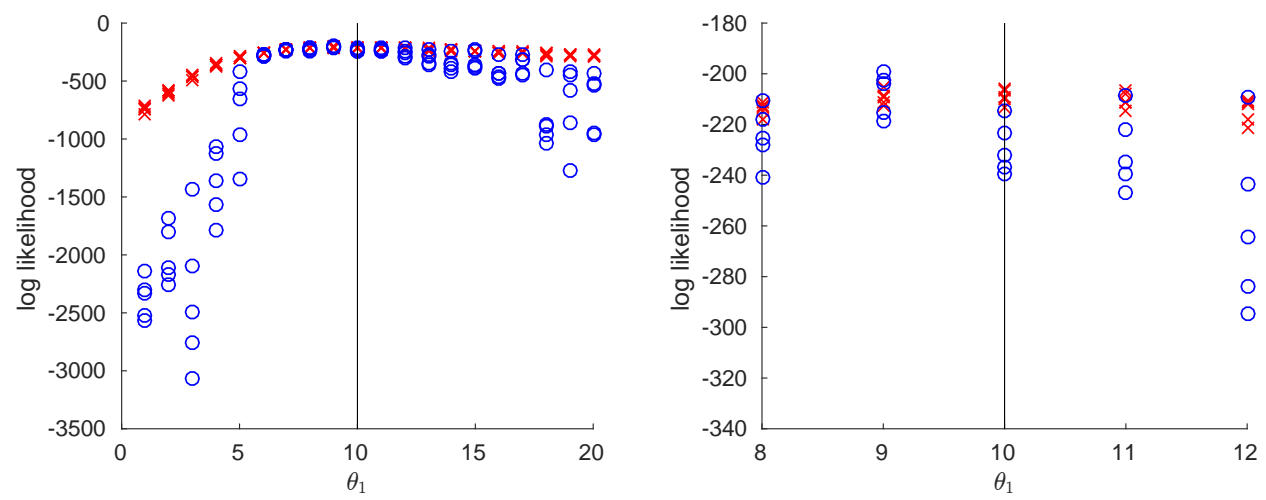

Figure 9: Lorenz 63 log likelihood estimates using the BPF (blue circles) and EnKF (red crosses) as $\theta_{1}$ is varied and the other parameters are held constant at their true values. Both plots show the same estimates, but the right hand plot zooms in to a smaller plot range. The vertical line shows the true parameter value. 


\section{Appendix D: Posterior estimates for Lorenz 96 example}
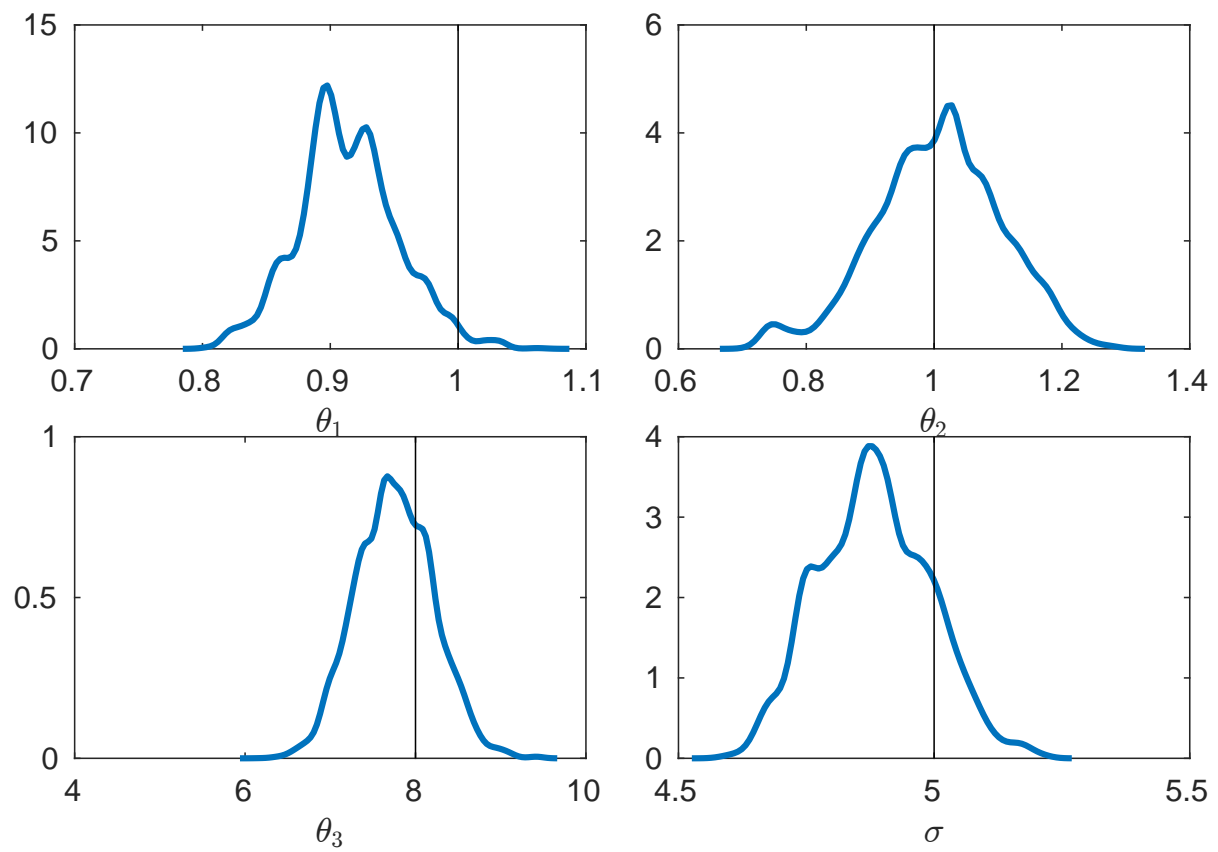

Figure 10: Estimated Lorenz 96 marginal parameter posteriors using eMCMC. These are kernel density estimates based on Monte Carlo samples. Vertical lines show the true parameter values. 


\section{Appendix E: Additional results for the Lotka-Volterra example}
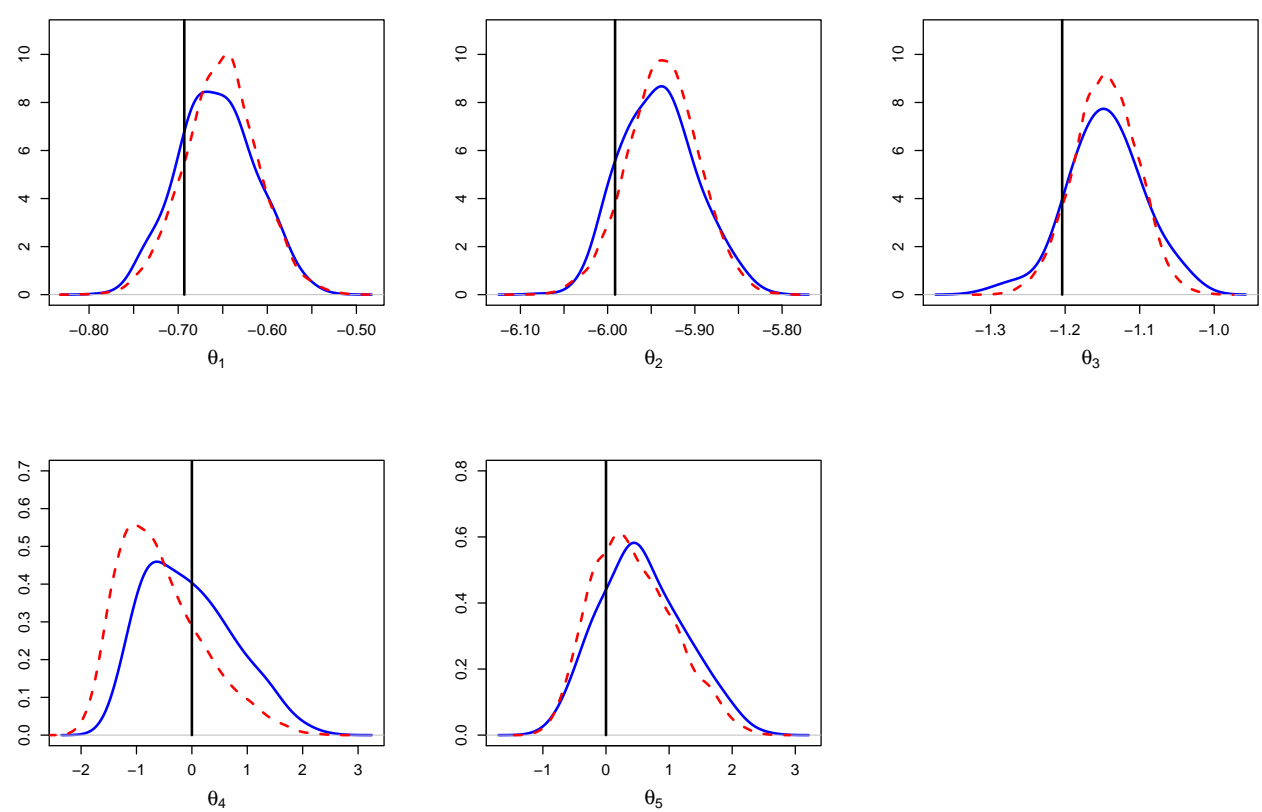

Figure 11: Lotka Volterra dataset $\mathcal{D}_{2}$. Marginal posterior densities based on the output of pMCMC (solid) and eMCMC (dashed). 

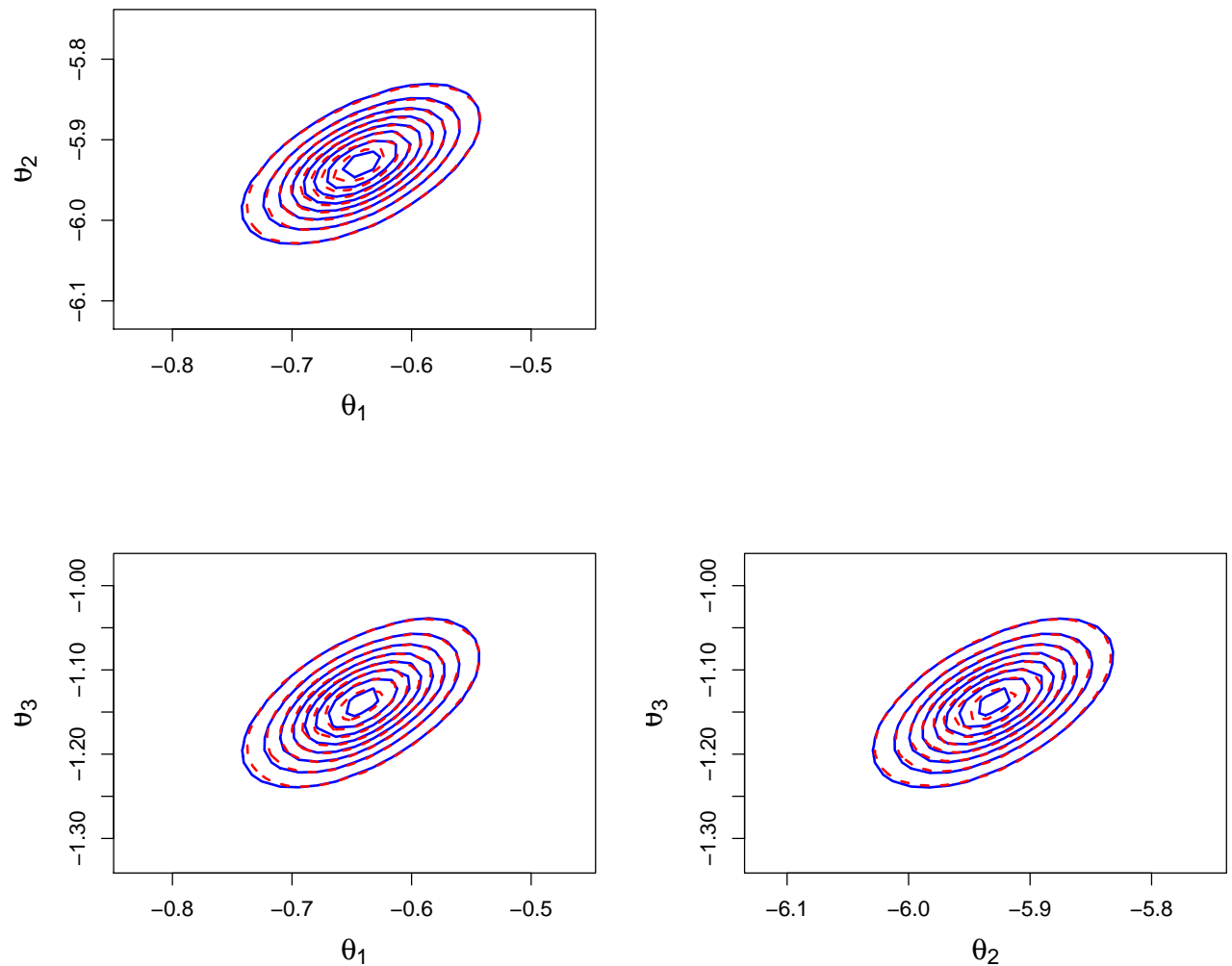

Figure 12: Lotka Volterra dataset $\mathcal{D}_{1}$. Bivariate posterior densities based on the output of pMCMC (solid) and eMCMC (dashed).

\section{Appendix F: Posterior estimate for datasets 2 of the Autoregulatory Network example}



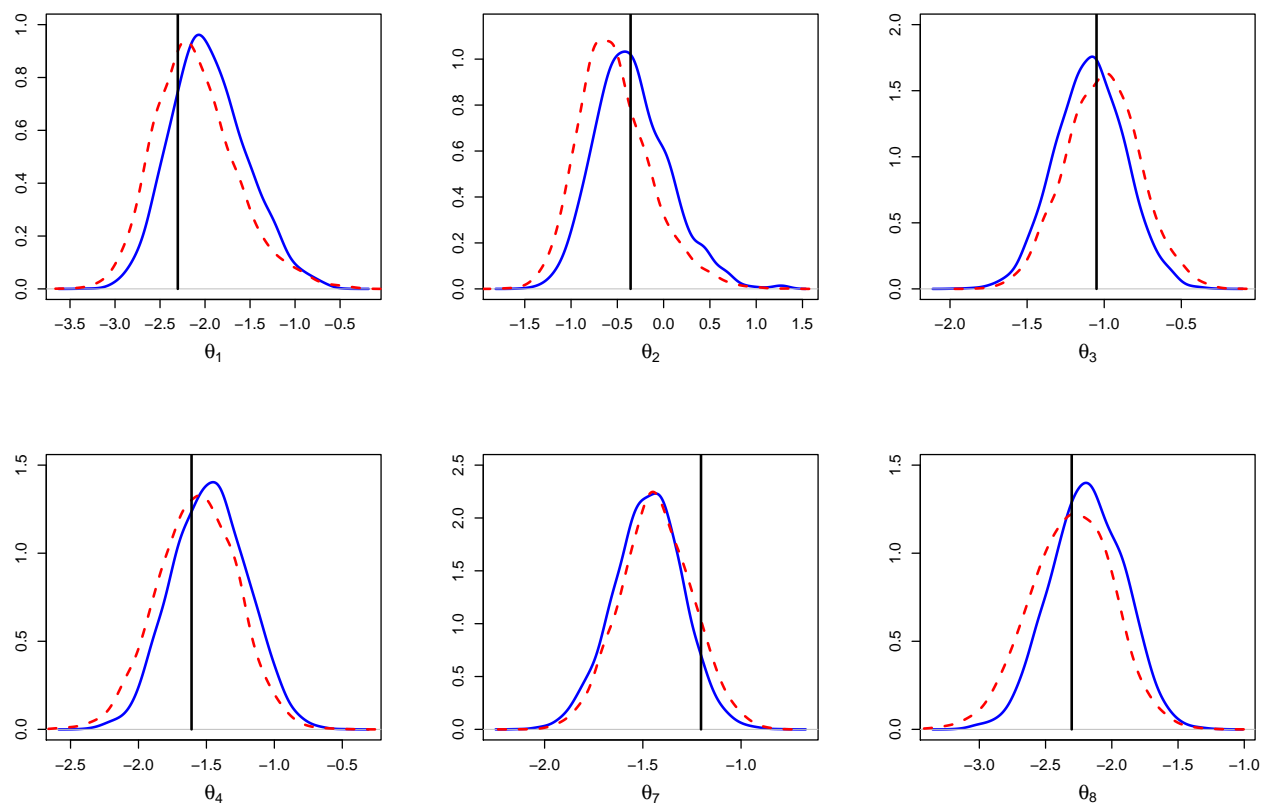

Figure 13: Autoregulatory dataset $\mathcal{D}_{2}$. Marginal posterior densities based on the output of pMCMC (blue solid) and eMCMC (red dash).

\section{Appendix G: Parameters for the Liley model}




\begin{tabular}{|c|c|c|}
\hline Parameter & Prior & Value for simulation \\
\hline \hline$\Gamma_{e e}$ & $\mathcal{U}(0.1,2)$ & 0.10631 \\
\hline$\Gamma_{e i}$ & $\mathcal{U}(0.1,2)$ & 0.64105 \\
\hline$\Gamma_{i e}$ & $\mathcal{U}(0.1,2)$ & 0.46477 \\
\hline$\Gamma_{i i}$ & $\mathcal{U}(0.1,2)$ & 0.28663 \\
\hline$\gamma_{e e}$ & $\mathcal{U}(100,1000)$ & 291.5 \\
\hline$\gamma_{e i}$ & $\mathcal{U}(100,1000)$ & 697.76 \\
\hline$\gamma_{i e}$ & $\mathcal{U}(10,500)$ & 458.67 \\
\hline$\gamma_{i i}$ & $\mathcal{U}(10,500)$ & 82.33 \\
\hline $\bar{p}_{e e}$ & $\mathcal{U}(0,10000)$ & 6603.4 \\
\hline $\bar{p}_{e i}$ & $\mathcal{U}(0,10000)$ & 2625.7 \\
\hline$\sigma$ & $\mathcal{U}(0,10)$ & 0.01 \\
\hline
\end{tabular}

Table 1: Parameters of the Liley model. 